\title{
Parallel processing of quickly and slowly mobilized reserve vesicles in hippocampal synapses
}

\author{
Kashif Mahfooz ${ }^{1}$, Mathan K. Raja², John F. Wesseling ${ }^{3, *}$ \\ 1 Dept. of Pharmacology, University of Oxford, UK \\ 2 Dept. Neurociencias (CIMA), Universidad de Navarra, Pamplona, Spain \\ 3 Institute for Neurosciences CSIC-UMH, San Juan de Alicante, Spain \\ * john.wesseling@csic.es
}

\begin{abstract}
Quickly and slowly mobilized reserve vesicles within presynaptic terminals are thought to be contained within separate pools that are connected in series. However, here we use FM-dyes to show that the two types are mobilized in parallel, without intermixing. The result supports a re-conceptualization of synaptic vesicle trafficking, proposed previously, where: (1) active zones contain multiple independent docking/release sites; (2) the release sites vary in probability of catalyzing exocytosis following individual action potentials; and (3), each docked vesicle is connected to a separate reserve. The re-conceptualization is then supported further by evidence that alterations in the timing of reserve pool depletion in synapsin knockouts are largest during lightest use even though alterations in short-term synaptic plasticity are largest during heavy use. The re-conceptualization implies that low release probability sites account for both reluctant readily releasable vesicles and slowly mobilized reserves. Extensive heterogeneity suggests that synapses have the capacity to store information by modulating the ratio of low to high probability release sites.
\end{abstract}

\section{Introduction}

Chemical synapses often exhibit striking dynamic changes in connection strength during the type of repetitive use that occurs regularly in vivo. The changes are termed short-term plasticity or frequency dynamics (Tsodyks and Markram, 1997; Abbott and Regehr, 2004; Buonomano and Maass, 2009). Multiple presynaptic vesicle trafficking mechanisms are involved, but the identity of the mechanisms, how they interact, and the implications for biological computation are not understood (Neher, 2015).

Most attempts at a higher resolution understanding have subdivided presynaptic vesicles into multiple pools, including: a small readily releasable pool subdivided further into fast-releasing and reluctant/slow-releasing components (Wu and Borst, 1999; Sakaba and Neher, 2001; Moulder and Mennerick, 2005); and multiple reserve pools that are likewise distinguished from each other by how quickly the contents are mobilized for release during electrical stimulation (Neves and Lagnado, 1999; Sakaba and Neher, 2001; Moulder and Mennerick, 2005; Rizzoli and Betz, 2005; Denker et al., 2011). A

widespread premise has been that the pools are connected in series (see Figure 1A), and that net flow between them is influenced by the number of constituents of each by the principle of mass action (Denker and Rizzoli, 2010; Rothman et al., 2016; Lee et al., 
2013; Miki et al., 2016; Doussau et al., 2017; Milovanovic et al., 2018). However, accumulating evidence argues against this premise, both for readily releasable and reserve pools.

That is: A variety of techniques have been used recently to show that vesicles in the reluctantly-releasing subdivision of the readily releasable pool can undergo exocytosis directly, in parallel with the fast-releasing subdivision (Hu et al., 2013; Müller et al., 2015; Mahfooz et al., 2016; Böhme et al., 2016; Maschi and Klyachko, 2020; see figure 5-supplement 1 of Raja et al., 2019 for analysis of a key caveat proposed in Miki et al., 2016). The results suggest that reluctantly releasable vesicles are released slowly during trains of action potentials because they are docked at the plasma membrane to inefficient release sites that catalyze exocytosis with low probability following the individual action potentials, and not because they are instead in an immature state that is upstream of the fast-releasing subdivision of the readily releasable pool as previously thought.

Meanwhile, reserve vesicles are not docked, but are instead located in the interiors of presynaptic terminals, within nearby clusters termed clouds. Imaging experiments have not detected the type of diffusive motion within the clouds that would be expected of mass action mechanisms (Lemke and Klingauf, 2005; Gaffield and Betz, 2007; Guillaud et al., 2017). And, our own analysis of synaptic throughput during heavy electrical stimulation argued strongly against any influence of mass action mechanisms on rate-limiting vesicle trafficking steps, at least for excitatory hippocampal synapses (Stevens and Wesseling, 1999; Garcia-Perez et al., 2008).

More specifically, vesicle recruitment from the reserve to readily releasable pool seems to be rate-limited by biochemical priming that occurs after physical docking, and would therefore not be expected to be influenced by the mass action of reserve vesicles (Miki et al., 2020). And indeed, although our synaptic throughput experiments supported the general concept that recruitment slows down after depleting reserve pools, a detailed kinetic analysis indicated that the slow-down was not gradual as expected of mass action. Instead, the slow-down occurred because the timing of recruitment at individual release sites switched kinetic modes in an all-or-none fashion from normal operation to a mode where new vesicles are recruited $>10$-fold more slowly. And, both modes were operant in individual synapses at the same time (Garcia-Perez et al., 2008).

To explain the modal recruitment, we proposed a re-conceptualization - which we now term the modular model - where vesicles throughout the clouds would be linked together in short chains that could each dock to a release site as a modular unit (Gabriel et al., 2011; see Wesseling et al., 2019 for supporting morphological evidence). Docking would be via the first vesicle in the chain. Vesicles recruited from a chain that was already docked would account for the normal mode, whereas replacement of empty chains would account for the slow mode (see Figure 1B).

To our knowledge, no specific relationship has been proposed between reluctant readily releasable vesicles and slowly mobilized reserves. However, we now reason that, if the modular model is correct, reserve vesicles chained to reluctant readily releasable vesicles would appear to reside in a slowly mobilized reserve pool during low frequency stimulation because: (1) reserve vesicles would have to wait in line before becoming readily releasable until space became available at the release site; and, (2) space would become available more slowly on average at inefficient release sites because of the low probability of release following the individual action potentials. If so, the single concept of inefficient release sites might account for both reluctant readily releasable vesicles and slowly mobilized reserves. And, quickly and slowly mobilized reserves would be mobilized in parallel rather than in series as currently thought, and would therefore not intermix. We now confirm that slowly and quickly mobilized reserves do not intermix at hippocampal synapses, even during heavy stimulation, supporting the modular model 
and providing further evidence against core principles underlying competing ideas.

The present report additionally includes independent support for the modular model from a new kind of analysis of synapsin knockout synapses. Synapsin proteins are expressed in high numbers in presynaptic terminals and are heavily phosphorylated by activity-dependent intracellular signaling mechanisms (De Camilli et al., 1990). They have been widely hypothesized to play a variety of activity-dependent roles in mobilizing reserve vesicles for release (De Camilli et al., 1990; Evergren et al., 2007; Milovanovic et al., 2018) that are not compatible with the evidence against rate-limiting mass action mechanisms referenced above (see also Gaffield and Betz, 2007), or with the modular model. Because of this, we included an analysis of short-term synaptic plasticity at synapsin knockout synapses in Gabriel et al. (2011), where the modular model was first proposed. The analysis showed that the deficits seen in the knockouts could be fully explained by the modular model by reducing the length of the chains of vesicles without altering the values of the other three parameters. The prediction of reduced chain length in synapsin knockouts was subsequently confirmed with electron microscopy in Wesseling et al. (2019). We now reason that the modular model predicts that alterations in synapsin knockouts in the timing of depletion of reserve pools would be largest during light use, and substantially less prominent during heavy use, which we now confirm. The new analysis is notable because the deficits in short-term synaptic plasticity were instead largest during heavy use (Rosahl et al., 1995; Pieribone et al., 1995; Gabriel et al., 2011), and, to our knowledge, the modular model is the only model proposed so far that provides a straightforward explanation for the inverted symmetry.

\section{Results}

The first step was to confirm that hippocampal synapses in cell culture contain both quickly and slowly mobilized reserve pools. That is, the modular model was developed to account for matching results obtained in primary cell culture and in ex vivo slices, but cell cultures are better suited for the FM-dye imaging technique used to distinguish between reserve pools.

We first loaded all recycling vesicles with the FM4-64 dye (Betz et al., 1992; Chi et al., 2001). We used $60 \mathrm{~s}$ of $20 \mathrm{~Hz}$ electrical stimulation (1200 pulses) to drive vesicle recycling in the presence of FM4-64 (Ryan and Smith, 1995; Gaffield and Betz, 2006). We then removed the FM4-64 and monitored destaining caused by exocytosis during much slower $1 \mathrm{~Hz}$ stimulation lasting $15 \mathrm{~min}$.

The simplest models involving only a single reserve pool predict that each action potential would cause the terminals to destain by a constant fraction, and the overall time course of destaining would follow a single exponential function of the form:

$$
F(t)=\left(F_{0}-F_{\infty}\right) \cdot e^{\frac{-t}{\tau}}+F_{\infty}
$$

where $F$ is fluorescence intensity, $t$ is time, $\tau$ is the time constant, $F_{0}$ is the intensity before destaining and $F_{\infty}$ is the residual intensity after destaining with $20 \mathrm{~Hz}$ stimulation at the end of each experiment. Instead, as expected of multiple reserve pools, we found that destaining did not follow Eqn 1 for any parameter values; the first part of the time course was fit with Eqn 1 when $\tau=6.7 \mathrm{~min}$, but later parts diverged from this equation (Figure 2A).

To quantify the deviation from Eqn 1, we calculated the fractional destaining during each 2 min interval, each corresponding to 120 pulses of stimulation. Specifically, fractional destaining was defined as

$$
\Delta F_{i n t}=\frac{\Delta F}{F^{\prime}-F_{\infty}}
$$


where $F^{\prime}$ was the fluorescence intensity from the first image in each interval and $F_{\infty}$ was the background as in Eqn 1 - int signifies internal, to emphasize that $F^{\prime}$ is within the 2 min interval, and is therefore only equal to $F_{0}$ for the first interval.

When destaining is quantified this way, single exponential decay typified by Eqn 1 always produces a constant value (magenta open circle denoted by single reserve in Figure 2B); i.e., because the first derivative of an exponential function is always a closely related exponential function with the same time constant, and fractional destaining is essentially the ratio between the first derivative and the equation itself. The approximately 4 -fold difference between the $2^{\text {nd }}$ and $3^{\text {rd }}$ boxes in Figure $2 \mathrm{~B}$ therefore shows that fractional destaining decreased approximately 4-fold over the $15 \mathrm{~min}$ of $1 \mathrm{~Hz}$ stimulation.

Although the analysis in Figure 2A-B pertained to populations of synapses, the decrease in fractional destaining was not because some synapses destained more quickly than others. A striking amount of variation was indeed observed between individual synapses (Figure 2C-D; see also Waters and Smith, 2002 for a similar result, although interpreted differently). However, almost all individuals diverged from single exponentials, and Figure 2D shows that the modal behavior was similar to the summary statistics in Figure 2A.

Further experiments showed that the decrease in fractional destaining during $1 \mathrm{~Hz}$ stimulation was long lasting, with no recovery detected even after 8 min of rest (Figure 3).

Next, we evaluated the caveat that the decrease in fractional destaining might have been caused by long-term depression of neurotransmitter release, although this seemed unlikely because the experiments were done in the presence of glutamate receptor blockers. To evaluate the caveat, we measured destaining during $1 \mathrm{~Hz}$ stimulation after loading the FM4-64 with long trains of $1 \mathrm{~Hz}$ stimulation - which would have induced the long-term depression - instead of the $20 \mathrm{~Hz}$ stimulation used above. Destaining was not slower - indeed, it was faster $\left(2^{\text {nd }}\right.$ vs $1^{\text {st }}$ box in Figure 4 ) - ruling out long-term depression; see Legend of Figure 5 for why faster destaining after loading at $1 \mathrm{~Hz}$ is expected of most models where reserve vesicles are segregated into multiple pools.

Taken together, the results confirm the presence of quickly and slowly mobilized reserves within individual hippocampal synapses that do not intermix quickly, if at all, at least not during low frequency stimulation or during rest intervals. The lack of intermixing is in-line with the studies showing a lack of vesicle mobility referenced in the Introduction, and with an electron microscopy study showing only limited mixing over longer periods (Rey et al., 2015).

\section{No interaction between fractional destaining at $20 \mathrm{~Hz}$ vs at $1 \mathrm{~Hz}$}

For the remainder of this report, we refer to the concept of probability of release for individual release sites when occupied by a vesicle with $p_{r s}$ for probability of release per release site, which - for the reasons given in the Introduction - we currently believe is likely closely related to the concept of probability of release per vesicle within the readily releasable pool denoted by $p_{v}, \bar{P}_{V E S}$, or fusion efficiency in our previous reports. Note that both concepts are different in important ways from the concept of probability of release per synapse, sometimes denoted by $P_{r}$, which would depend on the summed $p_{r s}$ of all release sites in a synapse as well as occupancy (e.g., see Neher, 2017).

The modular model would ascribe the decrease in fractional destaining observed above during the first $12 \mathrm{~min}$ of $1 \mathrm{~Hz}$ stimulation to selective depletion of dye from readily releasable and reserve vesicles chained to release sites with high $p_{r s}$, whereas vesicles chained to low $p_{r s}$ sites would undergo exocytosis at a lower rate, causing the $>4$-fold slower phase of destaining observed at later times (see Figure 5A). The absence of recovery in fractional destaining during 8 min of rest in Figure 3 is 
consistent with the modular model, but slow recovery taking minutes would also have been consistent because slow spontaneous reversal of chain docking - which is predicted in combination with subsequent mixing of undocked chains - which is not predicted per $s e$, but would be consistent with previous results - would have left room for this (see Discussion). In any case, the results do not, by themselves, rule out sequential models where vesicles in the slowly mobilized reserve transit through the quickly mobilized reserve during high frequency stimulation before being recruited to the readily releasable pool (see Figure 5B), and further tests were needed for disambiguation.

To develop an assay that could distinguish between the modular model and sequential models, we started with the realization that the modular model predicts that reserve vesicles tethered to vesicles docked at low $p_{r s}$ sites would only be slow to mobilize during low frequency stimulation. In contrast, both low and high $p_{r s}$ release sites would be vacant most of the time when stimulation is $20 \mathrm{~Hz}$ or faster because $20 \mathrm{~Hz}$ is intense enough to drive the readily releasable pool to a nearly completely exhausted steady state (Wesseling, 2019), and the overall timing of vesicular exocytosis would therefore no longer be influenced by the mechanism that determines the value of $p_{\text {rs }}$ (Figure 6A, left two panels; see Raja et al., 2019 for proof of this principle specifically using FM4-64). Instead, the timing during $20 \mathrm{~Hz}$ stimulation is determined by the upstream rate-limiting mechanism whereby vesicles are recruited to the release sites, which is thought either to be similar at low and high $p_{r s}$ release sites (Wesseling and Lo, 2002; Garcia-Perez and Wesseling, 2008; Mahfooz et al., 2016), or actually faster at low $p_{\text {rs }}$ release sites (Sakaba and Neher, 2001). Therefore, the modular model predicts that the distinction between quickly and slowly mobilized reserves would disappear when stimulation is increased to $20 \mathrm{~Hz}$.

Sequential models would also predict that the distinction between quickly and slowly mobilized reserves would lessen or disappear during high frequency stimulation if the mechanism that causes mixing between the two types were accelerated by activity. Such activity-dependent intermixing has been proposed and attributed to phosphorylation of synapsin proteins (De Camilli et al., 1990; Evergren et al., 2007; Milovanovic et al., 2018). Notably, however, key elements of the idea specific for synapsin proteins were ruled out in our previous electrophysiological analysis of synapsin knockout synapses (Gabriel et al., 2011; see also Gaffield and Betz, 2007). And, the distinction between quickly and slowly mobilized reserves observed during low frequency stimulation is maintained in the knockouts (Supplementary Figures 1 \& 2 ). In any case, the modular model is unique in predicting that the distinction between quickly and slowly mobilized reserves would disappear rapidly, in the time it takes to exhaust the readily releasable pool, and then reappear during subsequent rest intervals after new vesicles were recruited to the vacant release sites (Figure 6A). In contrast, the sequential models that have been proposed would, to our knowledge, predict that intermixing would be much slower. And, all sequential models would have to predict that, once mixed, the two types of reserves would not rapidly unmix, so fractional destaining upon returning to low frequency stimulation would revert to the greater value seen at newly stained synapses.

Therefore, to disambiguate between the modular model and sequential models, we first compared the time courses of destaining during $20 \mathrm{~Hz}$ stimulation after $1 \mathrm{~Hz}$ trains of $0,5,10$, and $25 \mathrm{~min}$ (Figure 6B). As predicted by the modular model, no differences were detected (Figure $6 C$ ), even though fractional destaining had decreased 4.3 -fold to $23 \pm 11 \%$ of the initial value by the $25^{\text {th }}$ min of $1 \mathrm{~Hz}$ stimulation (Figure $6 D, 5^{\text {th }}$ box). This result argues for the modular model and against sequential models, which would have predicted slower destaining over the initial interval during $20 \mathrm{~Hz}$ stimulation that followed long trains of $1 \mathrm{~Hz}$ stimulation because of the time needed to thoroughly mix quickly and slowly mobilized reserves. 
To evaluate the caveat that the initial interval was too short to be detected by our measurement technique, we partially destained terminals with 4 s-long bursts of $20 \mathrm{~Hz}$ stimulation ( 80 pulses) before or after the first of a pair of $1 \mathrm{~Hz}$ stimulation trains, as diagrammed atop Figure $7 A$ and $7 B$, respectively. Again as predicted by the modular model, fractional destaining during $20 \mathrm{~Hz}$ stimulation was not altered by $4 \mathrm{~min}$ of preceding $1 \mathrm{~Hz}$ stimulation (Figure $7 \mathrm{C}$ ).

Finally, even if $20 \mathrm{~Hz}$ stimulation were sufficient to completely mix the quickly and slowly mobilized reserves in less than $4 \mathrm{~s}$, the pools would then have remained mixed afterwards. However, this is ruled out by the further observation that the $20 \mathrm{~Hz}$ stimulation did not cause any recovery in the amount of fractional destaining measured when $1 \mathrm{~Hz}$ stimulation was resumed (compare Figure $7 B$ and $7 D$ and $2^{\text {nd }}$ and $4^{\text {th }}$ boxes of Figure $\boldsymbol{7 E}$ ).

Taken together, the results in Figures 2-7 thus support the modular model. The results could additionally be consistent with other models, yet to be proposed, where quickly and slowly mobilized reserve pools distinguished during low frequency stimulation are mobilized in parallel, and where differences in mobilization rates are no longer seen when the stimulation frequency is increased to $20 \mathrm{~Hz}$. However, the results seem to be incompatible with the widespread premise that slow and quickly mobilized pools of reserves are connected in series. Notably, the conclusion is based on a new line of reasoning that is independent of the already published results and supporting logic explained in the Introduction.

\section{Confirmation of anomalous light-use phenotype in synapsin knockouts}

Multiple studies have shown that short-term depression in synaptic strength is selectively increased during long compared to short trains of heavy stimulation at synapses where synapsin proteins have been eliminated or inactivated (Rosahl et al., 1995; Pieribone et al., 1995; Gabriel et al., 2011). Nevertheless, the modular model predicts that the situation would be inverted for measurements of the rate of FM-dye destaining. That is, the kinetic studies that motivated development of the modular model included a comparative analysis of Synapsin $1 \& 2$ double knockouts (hereafter, Synapsin DKO), and explained increased short-term depression with the prediction that the chains of vesicles would be shorter; specifically, the chains would have an average length of 2.6 in Synapsin DKO synapses and 4 in wildtype (WT) (Gabriel et al., 2011). Meanwhile, no difference was detected in the size of readily releasable pools, in-line with Gitler et al. (2008). Thus, Synapsin DKO and WT synapses stained with FM4-64 would release the same amount of dye during short trains that emptied the readily releasable pools, but that were not long enough to drive exocytosis of all of the vesicles from any of the docked chains. But, the amount of destaining when quantified as the fraction of total would appear to be more because of the shorter chains.

For example, a stimulus train that exhausted the readily releasable pool would destain 1 vesicle per chain - i.e., the docked vesicle - which would correspond to $38 \%$ of the dye contained within a chain in Synapsin DKO synapses $(1 / 2.6)$, but only $25 \%$ of the dye contained in a WT chain $(1 / 4)$, which is 1.5 -fold more. In-line with this prediction, we found that Synapsin DKO synapses destained $1.51 \pm 0.20$-fold more than WT synapses during the $4 \mathrm{~s}$ of $20 \mathrm{~Hz}$ stimulation used above (Figure $8 \mathrm{~A}$ ).

Importantly, the prediction of a 1.5-fold difference pertains specifically to situations where the stimulus train is too short to expend all of the vesicles within any chain in either Synapsin DKO or WT synapses. Otherwise, the total number of vesicles undergoing exocytosis would be fewer at Synapsin DKO synapses because the chains are shorter and would become exhausted sooner (see Figure 3C of Gabriel et al., 2011). 
And indeed, differences between Synapsin DKO and WT during long trains of $20 \mathrm{~Hz}$ stimulation were barely detectable (Figure $8 B$ ), in-line with previous observations for Synapsin DKO synapses during $4 \mathrm{~min}$ of $1 \mathrm{~Hz}$ stimulation (Figure 9A) $1.53 \pm 0.19$-fold more for Synapsin DKO when the frequency was $0.1 \mathrm{~Hz}$ (Figure 9B).

The new analysis of synapsin knockouts therefore supports the modular model further by correctly predicting: (1) the extent of the difference in fractional destaining between WT and Synapsin DKO synapses both during short trains of $20 \mathrm{~Hz}$ stimulation and during long trains of the lowest frequency stimulation; and (2) that the difference would be smaller during long trains of high frequency stimulation. Indeed, to our knowledge, the modular model is the only one proposed so far that provides an explanation for how the FM4-64 destaining results would be related to increases in short-term synaptic depression that, conversely, only emerge when stimulation is heavy rather than light.

\section{Discussion}

Synaptic vesicle trafficking in presynaptic terminals is central to brain function and is an increasingly important target of anti-epilepsy medicines (Lyseng-Williamson, 2011). A higher resolution understanding might generate insight into the first principles underlying biological computation, and might aid second generation drug discovery (García-Pérez et al., 2015).

Here we show that quickly and slowly mobilized reserve pools of vesicles in hippocampal synapses are mobilized in parallel, rather than in series as previously thought. The experiments were designed to be a new test for a non-standard model of vesicle trafficking - termed the modular model - that was proposed previously to explain results that that are not compatible with a nexus of current concepts that are not related to distinctions between reserve pools, as explained in the Introduction. As such, the new result provides independent/orthogonal support for the modular model.

Moreover, experiments showing that the modular model correctly predicts unanticipated features of synapsin knockout synapses that are most evident during light use provide a third type of independent support; see Wesseling et al. (2019) for a fourth type. When taken together, the results therefore seem to provide strong support for the modular model, or at least for a reconceptualization of synaptic vesicle trafficking that incorporates key elements such as: no rate-limiting role in vesicle trafficking for mass action mechanisms; and, parallel processing of multiple reserve pools.

\section{Cytoskeletal scaffold}

The absence of recovery in fractional destaining seen during $1 \mathrm{~Hz}$ stimulation even after 8 min of rest in Figure 3 was surprising because the modular model contains a slow mechanism for exchanging docked with non-docked chains of vesicles (see Figure 1B). One possibility is that the chains might be attached to a stable cytoskeletal scaffold that prevents intermixing (e.g., Siksou et al., 2007; Fernández-Busnadiego et al., 2010; Cole et al., 2016). 


\section{Spontaneous release and last-in/first-out cycling}

The experiments in the present study were designed to measure vesicle exocytosis involved in action potential evoked release, whereas transmitter released spontaneously is thought to be stored in a different class of vesicles (Kavalali, 2015). In addition, the present results do not rule out the possibility that other types of synapses additionally or instead harbor a variety of reserve pools that are connected in series (Neves and Lagnado, 1999; Richards et al., 2003). In particular, there is no contradiction with the concept of last-in/first-out cycling, where recently recycled vesicles can be recruited to the readily releasable pool more rapidly than vesicles that have been in the reserve pool for long periods (Rizzoli and Betz, 2004; Kamin et al., 2010). For example, currently available results do not yet specify how the chains of vesicles would be reconstructed from recycling membrane and moved into position. One possibility consistent with timing information in Rey et al. (2015) is that newly formed chains would be more likely to dock than chains formed at earlier times, owing to slow mixing of non-docked chains within the vesicle cluster over $10^{\prime}$ s of min.

\section{Relation to multiple classes of vesicles}

The present results are consistent with the possibility that the decision about whether a recycling vesicle will ultimately be targeted to a high or low $p_{r s}$ release site is made at the time of entry into the reserve pool. Alternatively, the parallel mobilization of slow and fast reserves might be analogous to parallel cycling of vesicles involved in spontaneous release, which would then require multiple classes of vesicles (Raingo et al., 2012). However, adding the concept of multiple classes of vesicles could not replace the requirement for multiple classes of release sites because, otherwise, the readily releasable pool would eventually fill with reluctantly releasable during low frequency stimulation, which would result in a long-lasting type of depression that was not seen in experiments designed to test this (see Figure 4). Multiple classes of vesicles without multiple classes of release sites is additionally incompatible with the results in Maschi and Klyachko (2020).

\section{Multiplexed frequency filtering}

Finally, the modular model explains slowly mobilized reserves as a logical consequence of low $p_{r s}$ release sites, with no obvious functional significance of their own. However, the heterogeneity among synapses in Figure 2 might nevertheless be relevant for biological computation.

That is, it has already been noted that low $p_{r s}$ release sites function as high-pass/low-cut frequency filters when transmitting information encoded within presynaptic spike trains, whereas high $p_{r s}$ release sites function as low-pass/high-cut filters (Mahfooz et al., 2016). The presence of multiple types of release sites would therefore endow synapses with a mechanism for selectively transmitting multiple types of frequency information while filtering out other types, which is analogous to the concept of multiplexing in digital information technology. The heterogeneity in Figure 2 suggests that hippocampal synapses express mechanisms for modulating multiplexing, which might provide a means for storing more information than modulating only the synaptic connection strength evident during low frequency use, as currently thought (Bartol et al., 2015). Notably, we previously observed extensive variation between calyx of Held synapses in the ratio of fast-releasing to reluctant readily releasable vesicles, which, when taken together with the present results, suggests that mechanisms for modulating multiplexing might be available at a wide range of synapse types (Mahfooz et al., 2016). 


\section{Methods and Materials}

Cell culture and imaging methods were already described in Raja et al. (2019). Briefly: Imaging was performed 14-21 days after plating at $0.25 \mathrm{~Hz}$ using a CCD camera, 20X objective, and green LED. Bathing solution was exchanged continuously during imaging at $0.2-0.5 \mathrm{ml} / \mathrm{min}$ within a low volume sealed chamber of $\sim 35 \mu \mathrm{l}$. Electrical stimulation was bipolar $(0.5 \mathrm{~ms}$ at $-30 \mathrm{~V}$ then $0.5 \mathrm{~ms}$ at $+30 \mathrm{~V})$ via two platinum electrodes built into the chamber. Neurotransmitter receptors were blocked with (in $\mu \mathrm{M})$ : picrotoxin (50); DNQX (10); and DL-APV (50). Other solutes were (in $\mathrm{mM}$ ): $\mathrm{NaCl}$ (118); $\mathrm{KCl}(2)$; $\mathrm{Ca}^{2+}(2.6) ; \mathrm{Mg}^{2+}$ (1.3); Glucose (30); and HEPES (25). Selection criteria: Experiments were conducted on fields of view that were much smaller than the entire coverslip, and were selected either before or after staining with FM4-64. Staining tended to be brighter for experiments selected afterwards, and destaining during $1 \mathrm{~Hz}$ stimulation seemed to be faster, suggesting a bias for synapses with higher probability of release. Therefore, only fields of view selected before staining were used when the staining protocol was varied experimentally (Figure 4 and Supplementary Figure $1 B$ ) and for quantitative comparisons between WT and synapsin DKO (Figures 8 \& 3 9). Processing: Time lapse images were aligned and Regions of Interest (ROIs) identified as described in Raja et al. (2019) (see Supplementary Figure 3). Between 175 and 1773 ROIs were detected for each field of view. For summary statistics, the median values from a single field of view/experiment were counted as $n=1$ unless otherwise indicated. Normalization: For comparing images across preparations, median or individual ROI values were: divided by the mean value of the background region; corrected for any rundown; and then normalized by $F_{0}$ in Eqn 1 as described in Raja et al. (2019).

362

\section{$5 \quad$ Funding}

This work was funded by: the Spanish Ministry of Science (BFU2009-12160 and SAF2013-48983R and BFU2016-80918-R) and the Unión Temporal de Empresas (UTE) project at the Centro de Investigación Médica Aplicada of the Universidad de Navarra. The funders had no role in study design, data collection and analysis, or preparation of the manuscript.

\section{Acknowledgments}

We thank Daniela Urribarri for technical assistance, Dr. Silvio Rizzoli for help understanding relationships between previous models of synaptic vesicle cycling, and Drs. Donald Lo, William Wetsel, Robert Renden, and Jay Coggan for advice about the writing.

\section{References}

Abbott LF, Regehr WG. Synaptic computation. Nature. 2004 oct; 431(7010):796-803. doi: 10.1038/nature03010.

Bartol TM, Bromer C, Kinney J, Chirillo MA, Bourne JN, Harris KM, Sejnowski TJ. Nanoconnectomic upper bound on the variability of synaptic plasticity. eLife. 2015 nov; 4:e10778. doi: 10.7554/eLife.10778. 
Betz WJ, Mao F, Bewick GS. Activity-dependent fluorescent staining and destaining ${ }_{402}$ of living vertebrate motor nerve terminals. Journal of Neuroscience. 1992; ${ }_{403}$ 12(February):363-375.

Böhme MA, Beis C, Reddy-Alla S, Reynolds E, Mampell MM, Grasskamp AT, $\quad{ }_{405}$ Lützkendorf J, Bergeron DD, Driller JH, Babikir H, Göttfert F, Robinson IM, ${ }_{406}$ O’Kane CJ, Hell SW, Wahl MC, Stelzl U, Loll B, Walter AM, Sigrist SJ. Active zone ${ }^{407}$ scaffolds differentially accumulate Unc13 isoforms to tune $\mathrm{Ca}(2+)$ channel-vesicle ${ }_{408}$ coupling. Nature Neuroscience. 2016 oct; 19(10):1311-20. doi: 10.1038/nn.4364. 409 Buonomano DV, Maass W. State-dependent computations: spatiotemporal 410 processing in cortical networks. Nature Reviews Neuroscience. 2009 feb; 10(2):113-25. ${ }^{411}$ doi: $10.1038 / \mathrm{nrn} 2558$.

Chi P, Greengard P, Ryan TA. Synapsin dispersion and reclustering during synaptic activity. Nature Neuroscience. 2001; 4(12):1187-93. doi: 10.1038/nn756.

Cole AA, Chen X, Reese TS. A Network of Three Types of Filaments Organizes Synaptic Vesicles for Storage, Mobilization, and Docking. Journal of Neuroscience. 2016; 36(11):3222-3230. doi: 10.1523/JNEUROSCI.2939-15.2016.

De Camilli P, Benfenati F, Valtorta F, Greengard P. The synapsins. Annual Review ${ }_{418}$ of Cell Biology. 1990; 6:433-60. doi: 10.1146/annurev.cb.06.110190.002245.

Denker A, Bethani I, Kröhnert K, Körber C, Horstmann H, Wilhelm BG, Barysch SV, Kuner T, Neher E, Rizzoli SO. A small pool of vesicles maintains synaptic activity in vivo. Proceedings of the National Academy of Sciences of the United States of America. 2011; 108(41):17177-82. doi: 10.1073/pnas.1112688108.

Denker A, Rizzoli SO. Synaptic vesicle pools: an update. Frontiers in synaptic ${ }_{424}^{424}$ neuroscience. 2010 jan; 2(October):135. doi: 10.3389/fnsyn.2010.00135.

Doussau F, Schmidt H, Dorgans K, Valera AM, Poulain B, Isope P. Frequency-dependent mobilization of heterogeneous pools of synaptic vesicles shapes presynaptic plasticity. eLife. 2017 oct; 6 . doi: 10.7554/eLife.28935.

Evergren E, Benfenati F, Shupliakov O. The synapsin cycle: A view from the synaptic endocytic zone. Journal of Neuroscience Research. 2007; 85(12):2648-2656. doi: $10.1002 / j n r .21176$.

Fernández-Busnadiego R, Zuber B, Maurer UE, Cyrklaff M, Baumeister W, Lučić V. Quantitative analysis of the native presynaptic cytomatrix by cryoelectron tomography. Journal of Cell Biology. 2010; 188(1):145-156. doi: 10.1083/jcb.200908082.

Gabriel T, García-Pérez E, Mahfooz K, Goñi J, Martínez-Turrillas R, Pérez-Otaño I, Lo DC, Wesseling JF. A new kinetic framework for synaptic vesicle trafficking tested in synapsin knock-outs. Journal of Neuroscience. 2011 aug; 31(32):11563-77. doi: 10.1523/JNEUROSCI.1447-11.2011.

Gaffield MA, Betz WJ. Imaging synaptic vesicle exocytosis and endocytosis with FM dyes. Nature Protocols. 2006; 1(6):2916-21. doi: 10.1038/nprot.2006.476.

Gaffield MA, Betz WJ. Synaptic vesicle mobility in mouse motor nerve terminals with and without synapsin. Journal of Neuroscience. 2007; 27(50):13691-13700. doi: 10.1523/JNEUROSCI.3910-07.2007.

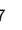

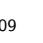


Garcia-Perez E, Lo DC, Wesseling JF. Kinetic isolation of a slowly recovering ${ }_{445}$ component of short-term depression during exhaustive use at excitatory hippocampal ${ }_{446}^{4}$ synapses. Journal of Neurophysiology. 2008 aug; 100(2):781-95. doi: 10.1152/jn.90429.2008.

García-Pérez E, Mahfooz K, Covita J, Zandueta A, Wesseling JF. Levetiracetam ${ }_{449}$ accelerates the onset of supply rate depression in synaptic vesicle trafficking. $\quad 450$ Epilepsia. 2015 apr; 56(4):535-45. doi: 10.1111/epi.12930.

Garcia-Perez E, Wesseling JF. Augmentation controls the fast rebound from ${ }_{452}$ depression at excitatory hippocampal synapses. Journal of Neurophysiology. 2008 apr; ${ }^{453}$ 99(4):1770-86. doi: 10.1152/jn.01348.2007.

Gitler D, Cheng Q, Greengard P, Augustine GJ. Synapsin IIa controls the reserve pool of glutamatergic synaptic vesicles. Journal of Neuroscience. 2008; 28(43):10835-43. doi: 10.1523/JNEUROSCI.0924-08.2008.

Guillaud L, Dimitrov D, Takahashi T. Presynaptic morphology and vesicular composition determine vesicle dynamics in mouse central synapses. eLife. 2017; 6:1-24. doi: 10.7554/eLife.24845.

Hu Z, Tong XJ, Kaplan JM. UNC-13L, UNC-13S, and Tomosyn form a protein code for fast and slow neurotransmitter release in Caenorhabditis elegans. eLife. 2013; 2013(2):1-20. doi: 10.7554/eLife.00967.

Kamin D, Lauterbach Ma, Westphal V, Keller J, Schönle A, Hell SW, Rizzoli SO. High- and low-mobility stages in the synaptic vesicle cycle. Biophysical Journal. 2010 jul; 99(2):675-84. doi: 10.1016/j.bpj.2010.04.054.

Kavalali ET. The mechanisms and functions of spontaneous neurotransmitter release. Nature Reviews Neuroscience. 2015 jan; 16(1):5-16. doi: 10.1038/nrn3875.

Lee JS, Ho WK, Neher E, Lee SH. Superpriming of synaptic vesicles after their recruitment to the readily releasable pool. PNAS. 2013 sep; 110(37):15079-84. doi: 10.1073/pnas. 1314427110 .

Lemke EA, Klingauf J. Single synaptic vesicle tracking in individual hippocampal boutons at rest and during synaptic activity. Journal of Neuroscience. 2005 nov; 25(47):11034-44. doi: 10.1523/JNEUROSCI.2971-05.2005.

Lyseng-Williamson KA. Levetiracetam: a review of its use in epilepsy. Drugs. 2011 mar; 71(4):489-514. doi: 10.2165/11204490-000000000-00000.

Mahfooz K, Singh M, Renden R, Wesseling JF. A Well-Defined Readily Releasable Pool with Fixed Capacity for Storing Vesicles at Calyx of Held. PLoS Computational Biology. 2016; 12(4):1-39. doi: 10.1371/journal.pcbi.1004855.

Maschi D, Klyachko VA. Spatiotemporal dynamics of multi-vesicular release is determined by heterogeneity of release sites within central synapses. eLife. 2020 feb; 9:1-20. doi: 10.7554/eLife.55210.

Miki T, Malagon G, Pulido C, Llano I, Neher E, Marty A. Actin- and Myosin-Dependent Vesicle Loading of Presynaptic Docking Sites Prior to Exocytosis. Neuron. 2016 aug; 91(4):808-23. doi: 10.1016/j.neuron.2016.07.033.

Miki T, Midorikawa M, Sakaba T. Direct imaging of rapid tethering of synaptic vesicles accompanying exocytosis at a fast central synapse. PNAS. 2020 jun; 117(25):14493-14502. doi: 10.1073/pnas.2000265117. 
Milovanovic D, Wu Y, Bian X, De Camilli P. A liquid phase of synapsin and lipid vesicles. Science. 2018 aug; 361(6402):604-607. doi: 10.1126/science.aat5671.

Moulder KL, Mennerick S. Reluctant vesicles contribute to the total readily releasable pool in glutamatergic hippocampal neurons. Journal of Neuroscience. 2005 apr; 25(15):3842-50. doi: 10.1523/JNEUROSCI.5231-04.2005.

Müller M, Genç Ö, Davis GW. RIM-binding protein links synaptic homeostasis to the stabilization and replenishment of high release probability vesicles. Neuron. 2015; 85(5):1056-1069. doi: 10.1016/j.neuron.2015.01.024.

Neher E. Merits and Limitations of Vesicle Pool Models in View of Heterogeneous Populations of Synaptic Vesicles. Neuron. 2015; 87(6):1131-1142. doi: 10.1016/j.neuron.2015.08.038.

Neher E. Some Subtle Lessons from the Calyx of Held Synapse. Biophysical Journal. 2017; 112(2):215-223. doi: 10.1016/j.bpj.2016.12.017.

Neves G, Lagnado L. The kinetics of exocytosis and endocytosis in the synaptic terminal of goldfish retinal bipolar cells. The Journal of Physiology. 1999 feb; 515(1):181-202. doi: 10.1111/j.1469-7793.1999.181ad.x.

Pieribone VA, Shupliakov O, Brodin L, Hilfiker-Rothenfluh S, Czernik AJ, Greengard P. Distinct pools of synaptic vesicles in neurotransmitter release. Nature. 1995 jun; 375(6531):493-7. doi: 10.1038/375493a0.

Raingo J, Khvotchev M, Liu P, Darios F, Li YC, Ramirez DMO, Adachi M, Lemieux P, Toth K, Davletov B, Kavalali ET. VAMP4 directs synaptic vesicles to a pool that selectively maintains asynchronous neurotransmission. Nature Neuroscience. 2012 mar; 15(5):738-45. doi: 10.1038/nn.3067.

Raja MK, Preobraschenski J, Del Olmo-Cabrera S, Martinez-Turrillas R, Jahn R, Perez-Otano I, Wesseling JF. Elevated synaptic vesicle release probability in synaptophysin/gyrin family quadruple knockouts. eLife. 2019 may; 8. doi: 10.7554/eLife.40744.

Rey Sa, Smith Ca, Fowler MW, Crawford F, Burden JJ, Staras K. Ultrastructural and functional fate of recycled vesicles in hippocampal synapses. Nature Communications. 2015 aug; 6(May):8043. doi: 10.1038/ncomms9043.

Richards DA, Guatimosim C, Rizzoli SO, Betz WJ. Synaptic vesicle pools at the frog neuromuscular junction. Neuron. 2003 jul; 39(3):529-41.

Rizzoli SO, Betz WJ. The structural organization of the readily releasable pool of synaptic vesicles. Science. 2004 mar; 303(5666):2037-9. doi: 10.1126/science.1094682.

Rizzoli SO, Betz WJ. Synaptic vesicle pools. Nature Reviews Neuroscience. 2005 jan; 6(1):57-69. doi: 10.1038/nrn1583.

Rosahl TW, Spillane D, Missler M, Herz J, Selig DK, Wolff JR, Hammer RE, Malenka RC, Südhof TC. Essential functions of synapsins I and II in synaptic vesicle regulation. Nature. 1995 jun; 375(6531):488-93. doi: 10.1038/375488a0.

Rothman JS, Kocsis L, Herzog E, Nusser Z, Silver RA. Physical determinants of vesicle mobility and supply at a central synapse. eLife. 2016 aug; 5. doi: 10.7554/eLife.15133. 
Ryan TA, Smith SJ. Vesicle pool mobilization during action potential firing at 531 hippocampal synapses. Neuron. 1995 may; 14(5):983-9.

Sakaba T, Neher E. Calmodulin mediates rapid recruitment of fast-releasing synaptic vesicles at a calyx-type synapse. Neuron. 2001 dec; 32(6):1119-31.

Siksou L, Rostaing P, Lechaire JP, Boudier T, Ohtsuka T, Fejtová A, Kao HT, Greengard P, Gundelfinger ED, Triller A, Marty S. Three-dimensional architecture of presynaptic terminal cytomatrix. Journal of Neuroscience. 2007 jun; 27(26):6868-77. doi: 10.1523/JNEUROSCI.1773-07.2007.

Stevens CF, Wesseling JF. Identification of a novel process limiting the rate of synaptic vesicle cycling at hippocampal synapses. Neuron. 1999 dec; 24(4):1017-28. doi: $10.1016 / \mathrm{s} 0896-6273(00) 81047-8$.

Tsodyks MV, Markram H. The neural code between neocortical pyramidal neurons ${ }_{542}$ depends on neurotransmitter release probability. Proc Natl Acad Sci USA. 1997 jan; ${ }_{543}$ $94(2): 719-23$.

Waters J, Smith SJ. Vesicle pool partitioning influences presynaptic diversity and weighting in rat hippocampal synapses. Journal of Physiology. 2002; 541(Pt 3):811-23. doi: 10.1113/jphysiol.2001.013485.

Wesseling JF. Considerations for Measuring Activity-Dependence of Recruitment of Synaptic Vesicles to the Readily Releasable Pool. Frontiers in Synaptic Neuroscience. 2019 nov; 11. doi: 10.3389/fnsyn.2019.00032.

Wesseling JF, Lo DC. Limit on the role of activity in controlling the release-ready supply of synaptic vesicles. Journal of Neuroscience. 2002 nov; 22(22):9708-20.

Wesseling JF, Phan S, Bushong EA, Siksou L, Marty S, Pérez-Otaño I, Ellisman M. Sparse force-bearing bridges between neighboring synaptic vesicles. Brain Structure and Function. 2019 dec; 224(9):3263-3276. doi: 10.1007/s00429-019-01966-x.

Wu LG, Borst JGG. The reduced release probability of releasable vesicles during recovery from short-term synaptic depression. Neuron. 1999 aug; 23(4):821-32. 


\section{A Serial model (current concept)}

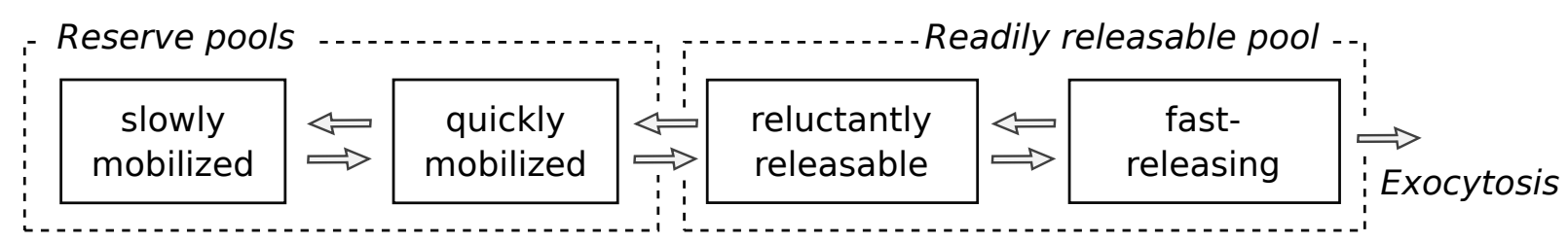

\section{B Modular model (re-conceptualization)}
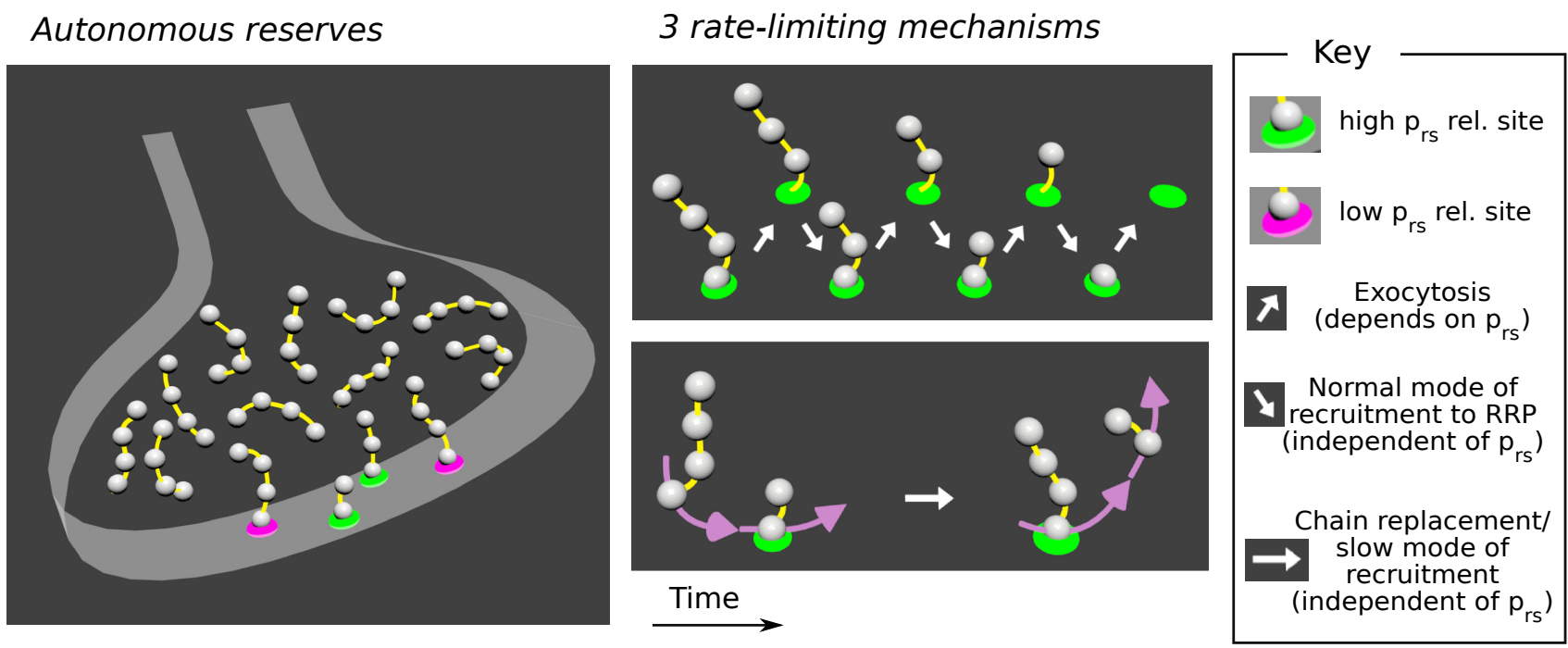

Figure 1. (A) The current concept is that reserve and readily releasable pools are connected in series. The diagram shows one version of this idea where transitions between each pool are reversible, but alternative variants have been proposed where some of the transitions only occur in a single direction. In addition, terminology varies between groups: e.g., slowly mobilized is referred to as reserve whereas quickly mobilized is recycling in Rizzoli and Betz (2005). (B) Modular model of rate limiting steps in synaptic vesicle trafficking. Vesicles throughout the cytoplasm are hypothesized to be linked together in short chains of 4 for WT and fewer for synapsin knockouts. Chains remain intact after a vesicle at one of the ends docks to a release site in the active zone and becomes ready for release. The other vesicles within the chain are then reserves, dedicated to resupplying the release site occupied by the docked vesicle when needed. Release sites are depicted as either green or magenta torus shapes; green represents efficient sites that catalyze exocytosis with a high probability (denoted as high $p_{r s}$ ) following an action potential, whereas magenta release sites catalyze exocytosis with low $p_{r s}$. The modular aspects of the model were first proposed in Gabriel et al. (2011). Variation among release sites in probability of release is based on results in Mahfooz et al. (2016); Raja et al. (2019); and Maschi and Klyachko (2020). See Wesseling et al. (2019) for morphological evidence for force-bearing bridges between vesicles that might be the links in the chains. 



Figure 2. Decelerating destaining rate during $1 \mathrm{~Hz}$ stimulation. (A) Destaining during $1 \mathrm{~Hz}$ stimulation (blue) followed by $20 \mathrm{~Hz}$ (red) as diagrammed at top. Black symbols are during intervals without stimulation. Plotted is the mean \pm s.e.m of the median values of regions of interest (ROIs) from $\mathrm{n}=12$ preparations: error bars are smaller than symbols; preparations had a mean of 715 ROIs, with a coefficient of variation of 0.48 The magenta curve is the single exponential that fits the first 2 min of destaining best, and has a time constant of $6.7 \mathrm{~min}$. See Methods and Materials for automatic detection of ROIs and for normalizing median values before averaging across preparations. (B) Comparison of fractional destaining (defined in Results) during the first and last 2 min of $1 \mathrm{~Hz}$ stimulation. Circles are median values; boxes demarcate the middle two quartiles of the distribution; *** signifies $\mathrm{p}<0.0005$ (Wilcoxon rank sum). The magenta circle is the prediction when intermixing is fast enough that destaining matches the exponential curve in Panel A. (C) Image of preparation stained with FM4-64. Scale bar is $20 \mu \mathrm{m}$. Insets are destaining of individual ROIs during the protocol diagrammed atop Panel A. Green lines are background. (D) Histogram of remaining fluorescence after $1 \mathrm{~Hz}$ stimulation for all individual ROIs from all 12 experiments in Panels A - C $(\mathrm{n}=8585$ ROIs $)$. 

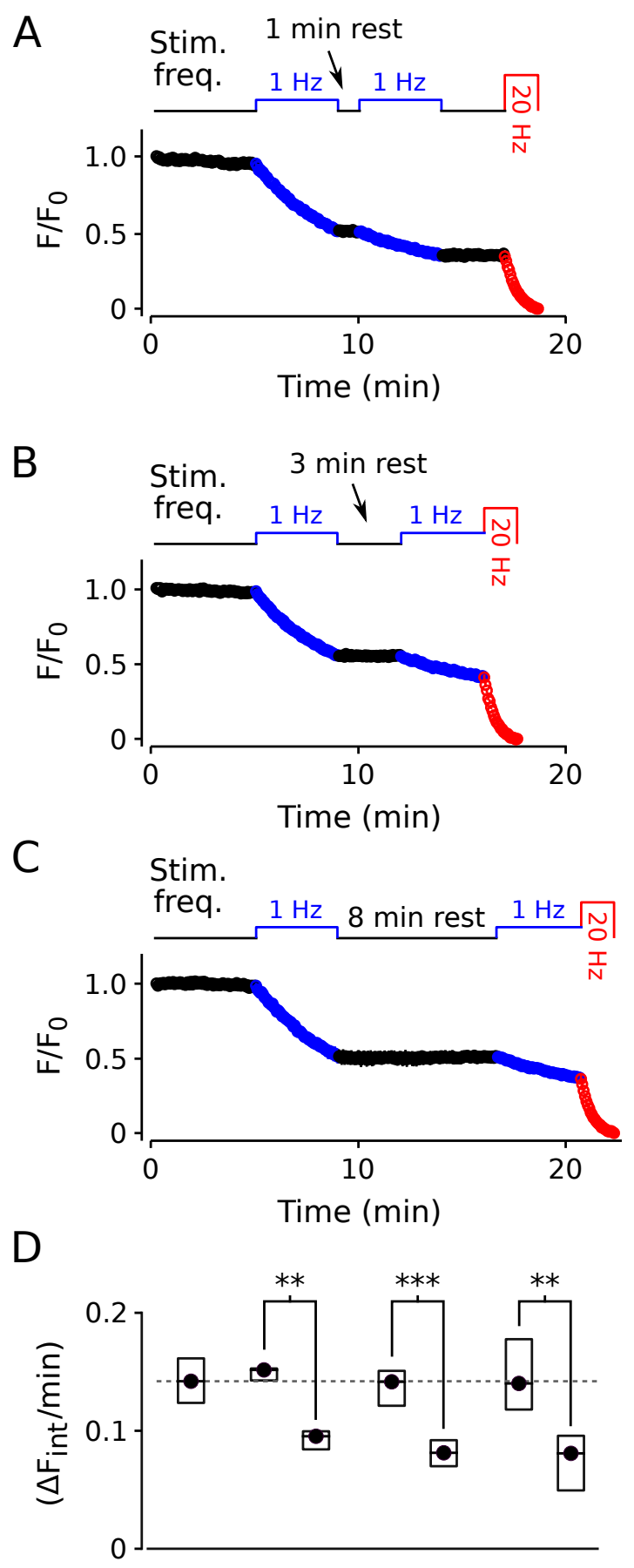

$1 \mathrm{~Hz}$ train $1^{\text {st }} \quad 1^{\text {st }} 2^{\text {nd }} \quad 1^{\text {st }} 2^{\text {nd }} \quad 1^{\text {st }} 2^{\text {nd }}$ rest
nterval all $1 \mathrm{~min} \quad 3 \mathrm{~min} \quad 8 \mathrm{~min}$

Figure 3. No evidence for intermixing even after $8 \mathrm{~min}$ of rest. Comparison of destaining during two trains of $1 \mathrm{~Hz}$ stimulation separated by $1 \mathrm{~min}$ for Panel A, $3 \mathrm{~min}$ for Panel B, and $8 \mathrm{~min}$ for Panel C. (D) Fractional destaining during: the first $2 \mathrm{~min}$ of the first and second $1 \mathrm{~Hz}$ trains as indicated; $\mathrm{n} \geq 5$ preparations; ${ }^{* *}, * * *$ signify $\mathrm{p}<0.01,0.001$ (Wilcoxon rank sum). 

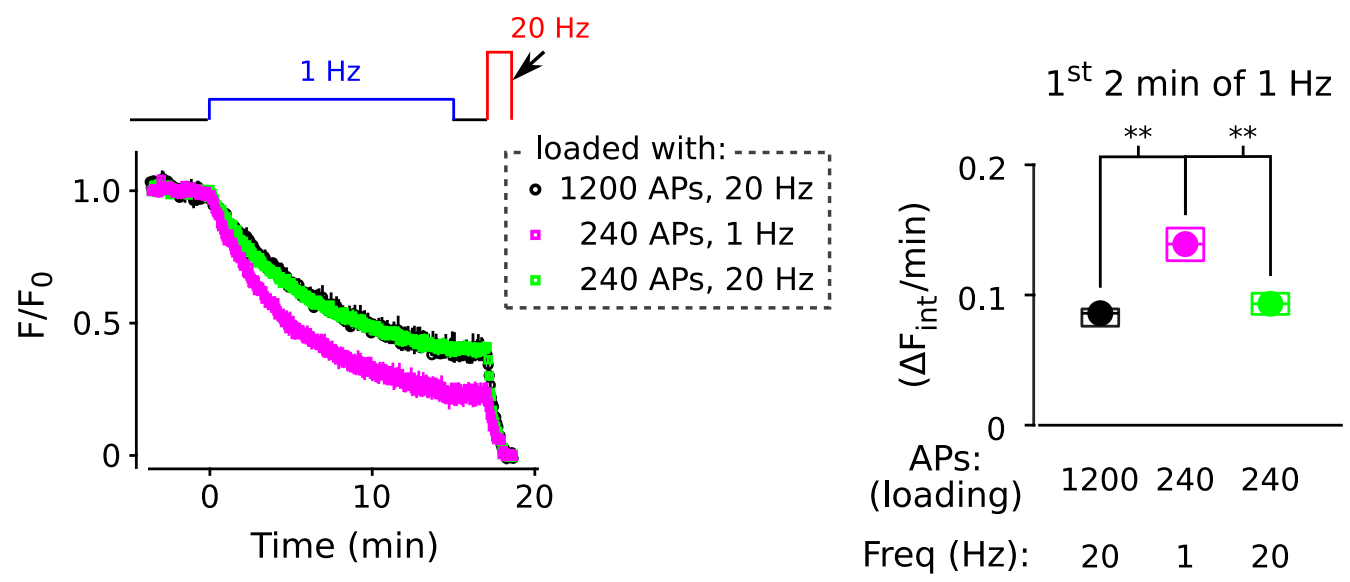

Figure 4. Faster destaining during $1 \mathrm{~Hz}$ stimulation when stimulation during the initial FM4-64 dye loading segment of the experiment was also at $1 \mathrm{~Hz} v s$ when at $20 \mathrm{~Hz}(\mathrm{n} \geq 4$ preparations; ${ }^{* *}$ signifies $\left.\mathrm{p}<0.01\right)$. Note that differences in destaining rates were not caused by differences in the amount of loading because destaining was equally slow when terminals were only partially loaded with short trains of $20 \mathrm{~Hz}$ stimulation $\left(3^{\text {rd }}\right.$ vs $1^{\text {st }}$ in boxplot). 

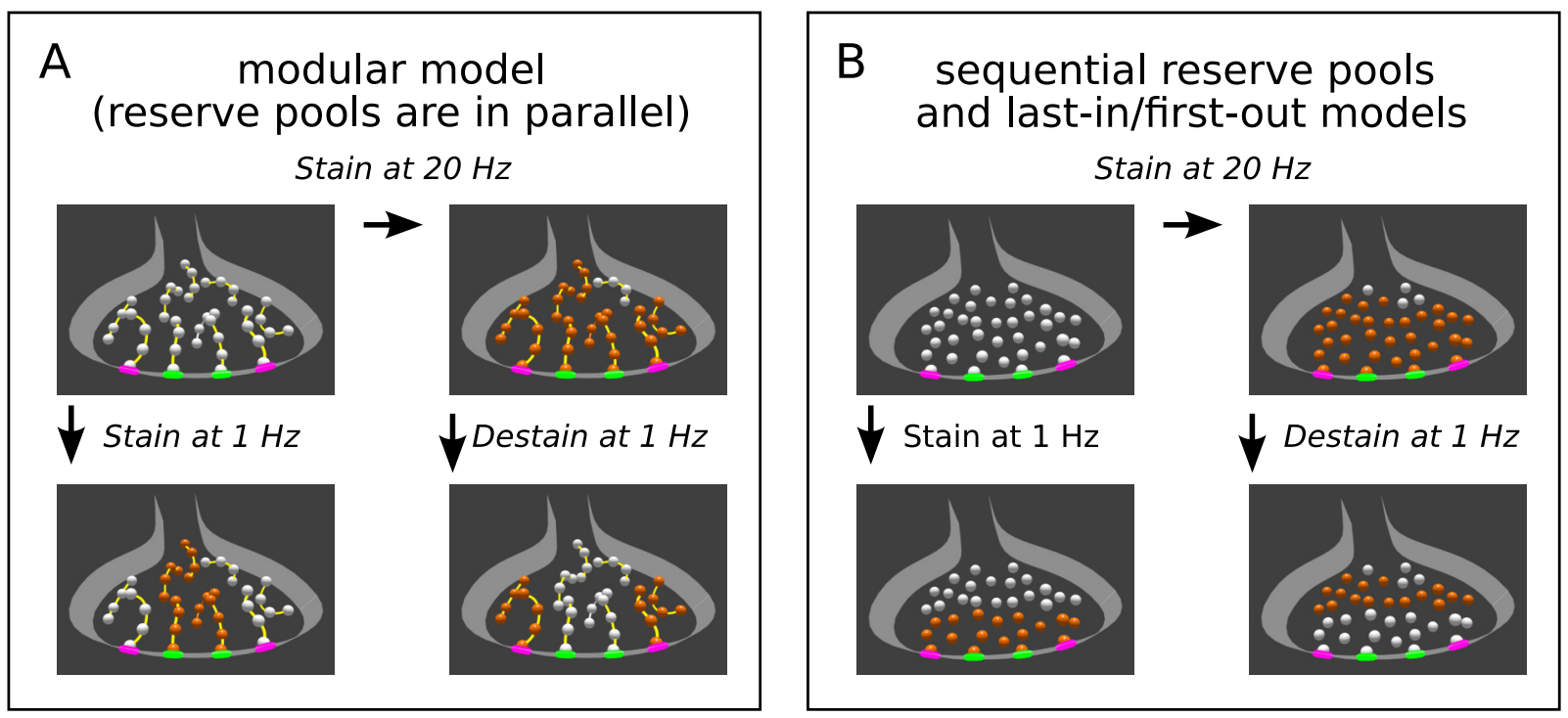

Figure 5. Comparison of modular model to previous models. Orange spheres are vesicles stained with FM4-64, gray are unstained. Green torus shapes are release sites with high $p_{r s}$, magenta have low $p_{r s}$. For all models, loading FM4-64 during $20 \mathrm{~Hz}$ stimulation stains all readily releasable and recycling reserve vesicles. Note that the illustrations are meant to highlight differences between the models and are not meant to be completely accurate; e.g., in reality, a much larger portion of the vesicles would be in a so called depot pool that does not participate in the exo/endoyctic cycle and is never stained (gray in upper right images in Panel A and Panel B).

Deviation from exponential: All models are compatible with the deviation from exponential decrease in fluorescence during $1 \mathrm{~Hz}$ stimulation in Figure 2, but the explanations are different. For the modular model: vesicles in chains docked at release sites with high $p_{\text {rs }}$ undergo exocytosis quickly compared to vesicles in chains that are docked at low $p_{r s}$ release sites (compare upper vs lower right images in Panel A). For sequential models, an easy to access reserve is mobilized first and destained quickly, leaving dye trapped in a deeper reserve that is mobilized slowly (compare upper vs lower right images in Panel B). For last-in/first-out models, vesicles destained during $1 \mathrm{~Hz}$ stimulation recycle quickly enough to occlude the release of the remaining stained vesicles.

Faster destaining when loading was at $1 \mathrm{~Hz}$ : Likewise, all models are compatible with the result in Figure 4 that destaining is faster during $1 \mathrm{~Hz}$ stimulation when FM4-64 was loaded with $1 \mathrm{~Hz}$ stimulation compared to when loaded with $20 \mathrm{~Hz}$. For the modular model, loading during $1 \mathrm{~Hz}$ stimulation selectively stains vesicles attached to release sites with high $p_{r s}$ (upper and lower left images in Panel A); fewer vesicles attached to low $p_{r s}$ release sites undergo exocytosis during the staining phase of the experiment, creating fewer vacancies for incorporating stained vesicles; this would be a consequence of the lack of mixing between reserves associated with high and low $p_{r s}$ release sites. For sequential models, loading during $1 \mathrm{~Hz}$ stimulation selectively stains the easy to access reserve pool, which is then released more quickly during subsequent $1 \mathrm{~Hz}$ stimulation. For last-in/first-out models, vesicles loaded during $1 \mathrm{~Hz}$ stimulation recycle quickly enough to occlude the release of unstained vesicles.

Support for modular model over alternatives: The modular model is distinguished experimentally from sequential and last-in/first-out models by comparing fractional destaining during $20 \mathrm{~Hz}$ stimulation initiated before $v s$ after partial destaining with $1 \mathrm{~Hz}$ stimulation; i.e. initiated from the starting points depicted by the upper vs lower right images for Panel $\mathbf{A}$ and Panel B; these results are in Figures $66 \%$. 
A

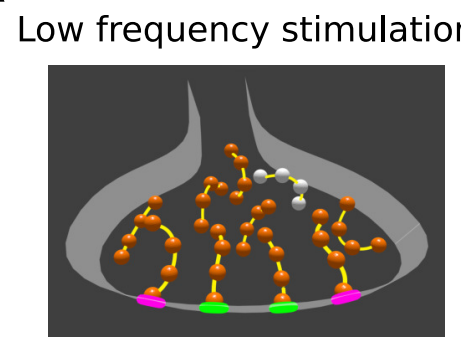

Release sites are occupied $p_{\text {rs }}$ determines rate of exocytosis

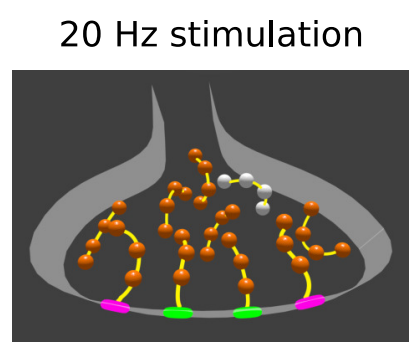

Release sites are vacant $p_{r s}$ is not relevant recruitment is rate-limiting

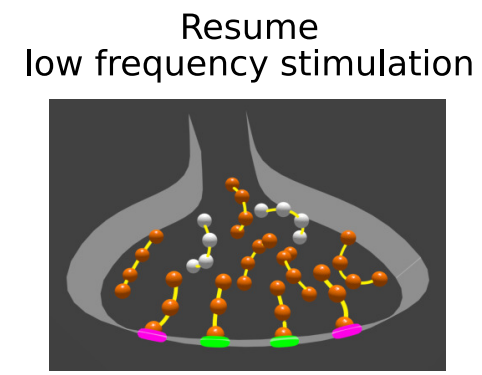

prs returns to relevance as soon as release sites are occupied

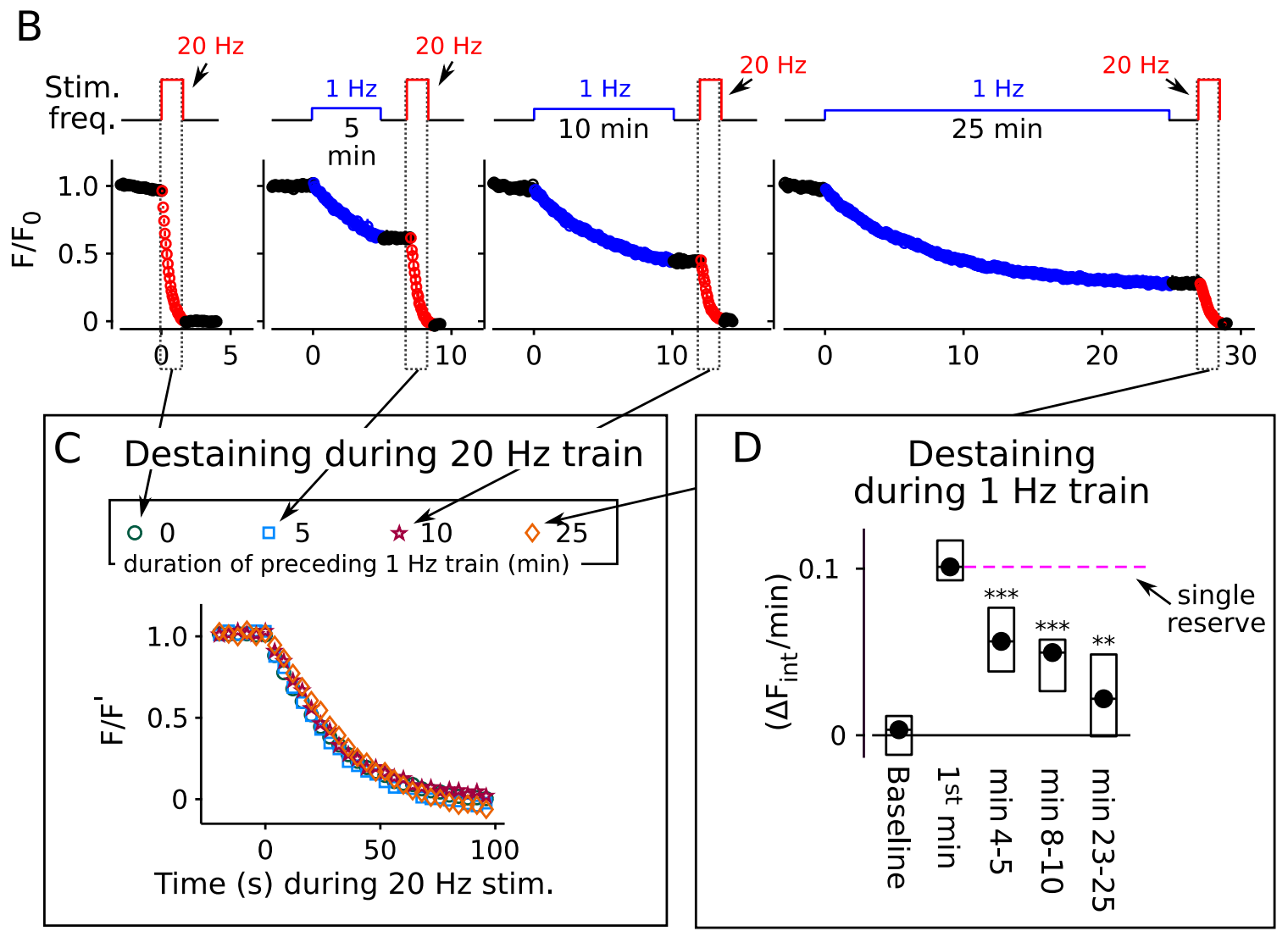

Figure 6. Partial destaining during $1 \mathrm{~Hz}$ stimulation does not alter the time course of destaining during subsequent stimulation at $20 \mathrm{~Hz}$. See Supplementary Figure 2 for the analogous experiment after knocking out synapsin $1 \& 2$. (A) Diagrams illustrating why $p_{r s}$ values influence the timing of destaining during low frequency stimulation, but not during trains of $20 \mathrm{~Hz}$ stimulation after the readily releasable pool has been exhausted. (B) Destaining during $100 \mathrm{~s}$ of $20 \mathrm{~Hz}$ stimulation following partial destaining by stimulating at $1 \mathrm{~Hz}$ for $0,5,10$, or $25 \mathrm{~min}(\mathrm{n} \geq 4$ preparations). (C) Overlay of time courses of destaining during $20 \mathrm{~Hz}$ stimulation. $F^{\prime}$ is mean fluorescence intensity during the 2 min rest intervals preceding $20 \mathrm{~Hz}$ stimulation. (D) Quantification of destaining during the $1 \mathrm{~Hz}$ trains (*** signifies $\mathrm{p}<0.001$ and ${ }^{* *}$ signifies $\mathrm{p}<0.005$, compared to destaining over the first 1 min of $1 \mathrm{~Hz}$ stimulation, Wilcoxon rank sum). 



Panel: A B
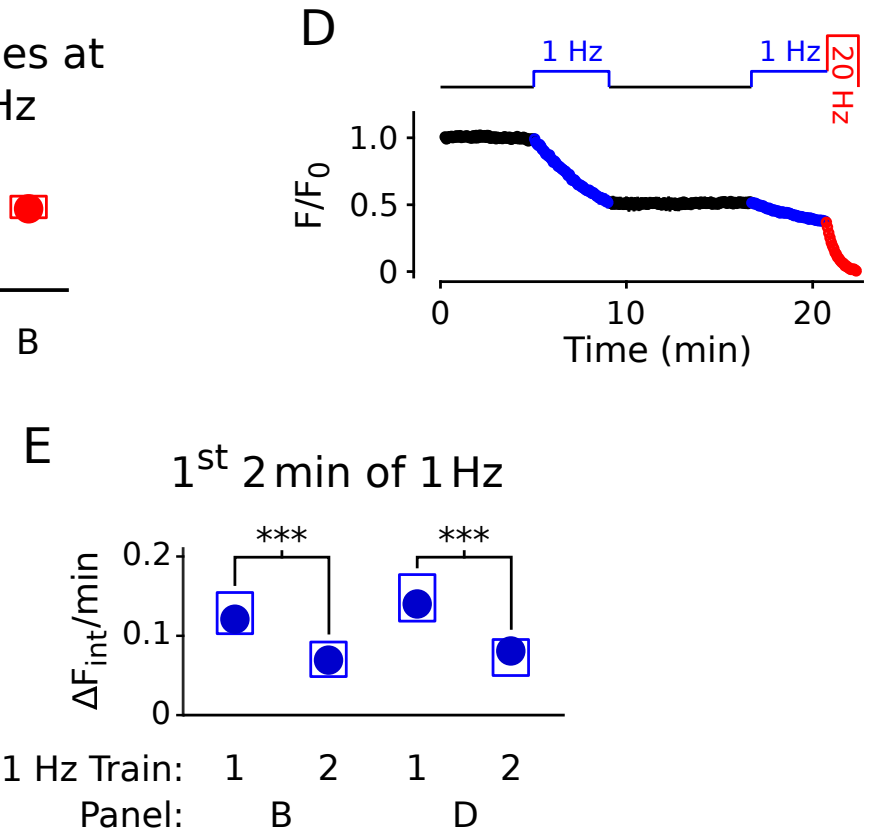

Figure 7. No interaction between deceleration in destaining during $1 \mathrm{~Hz}$ stimulation and destaining caused by short trains at $20 \mathrm{~Hz}$. (A) Destaining during $4 \mathrm{~s}$ of $20 \mathrm{~Hz}$ stimulation without preceding $1 \mathrm{~Hz}$ simulation. (B) Destaining during $4 \mathrm{~s}$ of $20 \mathrm{~Hz}$ stimulation after preceding $1 \mathrm{~Hz}$ simulation. (C) Quantification of fractional destaining during the 4 s-long trains of $20 \mathrm{~Hz}$ stimulation in Panels $\mathbf{A} \& \mathbf{B}$ ( $\mathrm{n} \geq 7$ preparations). (D) Identical to Panel B except without the $4 \mathrm{~s}$ train of $20 \mathrm{~Hz}$ stimulation. (E) The $4 \mathrm{~s}$ of $20 \mathrm{~Hz}$ stimulation in Panel B did not influence destaining during subsequent $1 \mathrm{~Hz}$ stimulation ( $\mathrm{n} \geq 6$ preparations; ${ }^{* * *}$ signifies $\mathrm{p}<0.005$, Wilcoxon rank rum). 

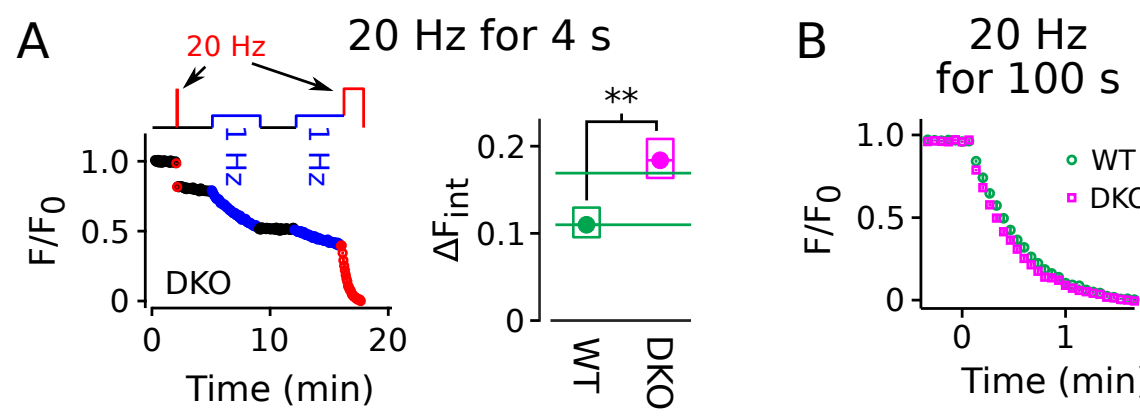

Figure 8. Accurate prediction of faster destaining in Synapsin DKO during short $20 \mathrm{~Hz}$ trains. (A) Quantification of amount of destaining during $4 \mathrm{~s}$ of $20 \mathrm{~Hz}$ stimulation; $\mathrm{n} \geq 4$ preparations. The experiment was designed to match the experiment already summarized in Figure 7A. ${ }^{* *}$ signifies p $<0.01$ (Wilcoxon rank sum). The green lines in boxplot are the predictions for DKO synapses derived by combining the median WT measurement with the 1.5-fold difference predicted by the modular model. (B) Comparison of time course of destaining during $100 \mathrm{~s}$ of $20 \mathrm{~Hz}$ stimulation confirming only minor differences between WT and Synapsin DKO for longer trains; data are from leftmost plots in Figure 6 and Supplementary Figure $2 A$. 


\section{A $\quad 1 \mathrm{~Hz}\left(1^{\text {st }} 4 \mathrm{~min}\right)$}
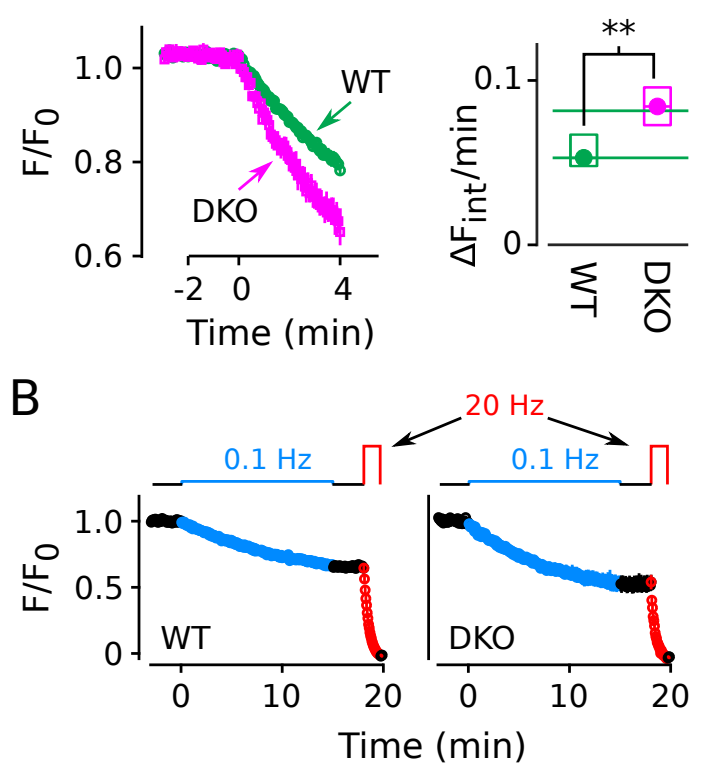

$1^{\text {st }} 4 \mathrm{~min}$ at $0.1 \mathrm{~Hz}$
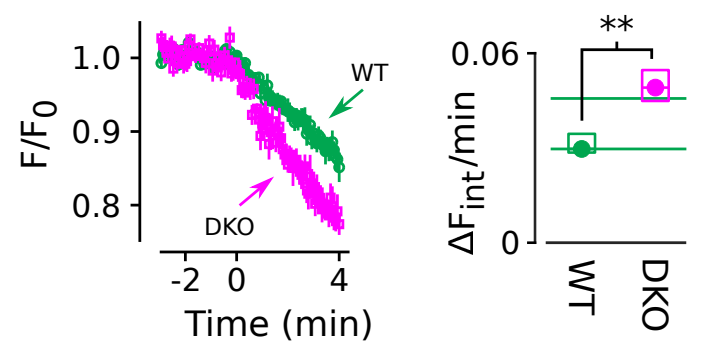

Figure 9. Correct prediction of faster destaining in Synapsin DKO during long low frequency trains. (A) Quantification of destaining rate during the first $4 \mathrm{~min}$ of $1 \mathrm{~Hz}$ stimulation; data are from rightmost plots in Figure 6 and Supplementary Figure 2A. (B) Quantification of destaining rate during the first 4 min of $0.1 \mathrm{~Hz}$ stimulation $(\mathrm{n}=6$ preparations). The green lines in boxplots are predictions for DKO synapses derived by combining the median WT measurement with the 1.5-fold difference predicted by the modular model $(* *, *$ signify $\mathrm{p}<0.01,0.05$, respectively (Wilcoxon rank sum). 


\section{Synapsin $1 \& 2$ DKO}
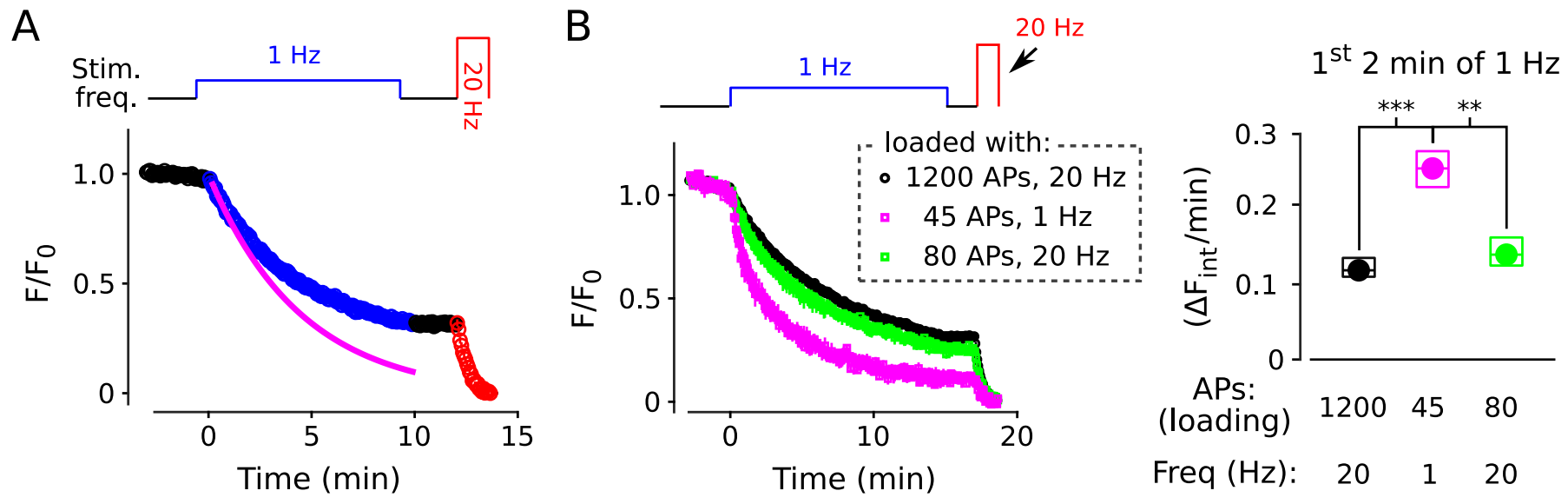

Supplementary Figure 1. Quickly and slowly mobilized reserves in Synapsin knockouts. (A) Analogous to Figure 2A; the magenta curve is Eqn 1 when $\tau=4.5 \mathrm{~min}$. (B) Analogous to Figure 4 ( $\mathrm{n} \geq 4$ preparations; ${ }^{* * *}$ signifies $\mathrm{p}<0.001,{ }^{* *}$ signifies $\mathrm{p}<0.01$, Wilcoxon rank sum). 


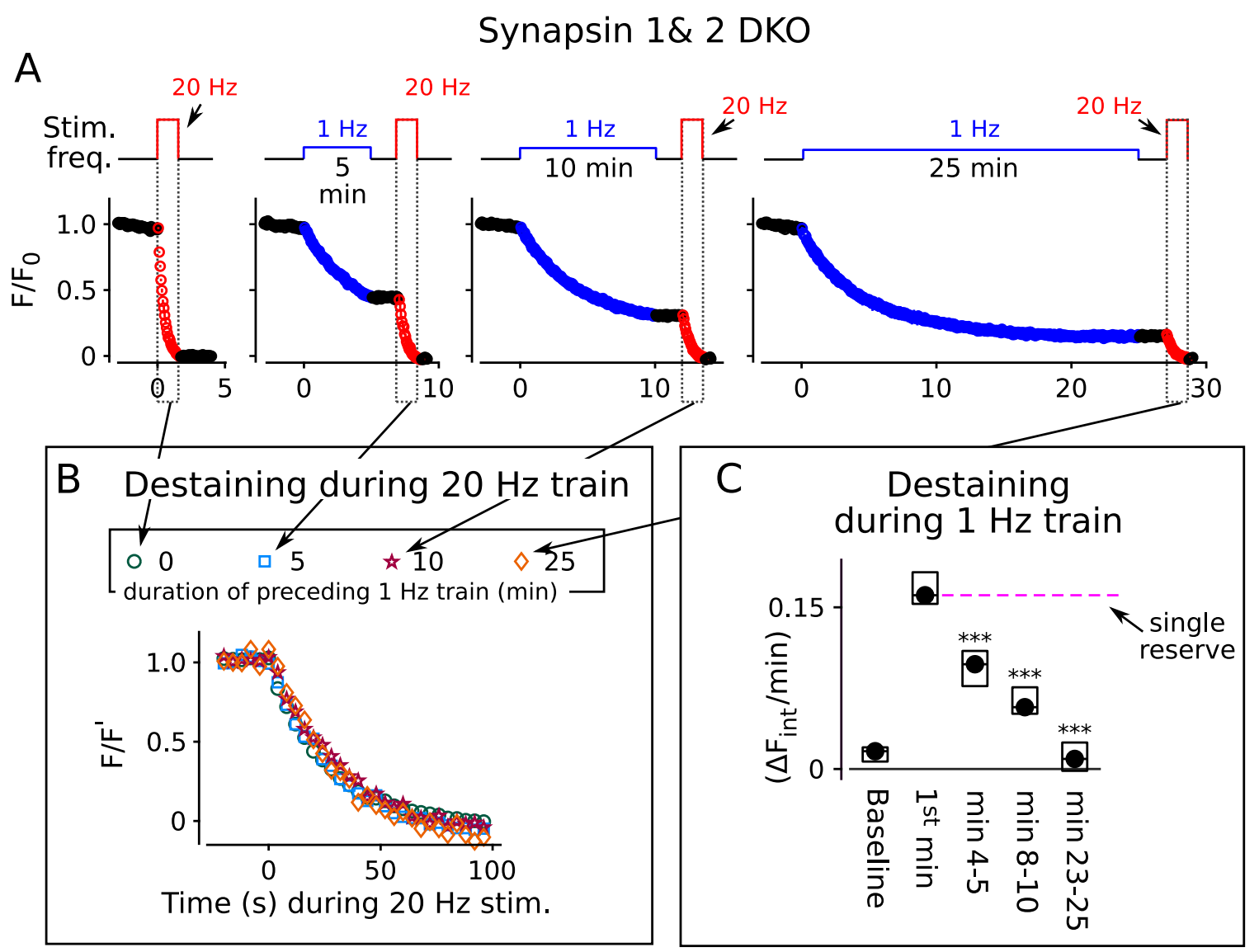

Supplementary Figure 2. Repeat of experiment in Figure 6 in Synapsin knockouts $(\mathrm{n} \geq 4$ preparations; ${ }^{* *}$ signifies $\mathrm{p}<0.001$ compared to destaining over the first $1 \mathrm{~min}$ of $1 \mathrm{~Hz}$ stimulation, Wilcoxon rank sum). 
A

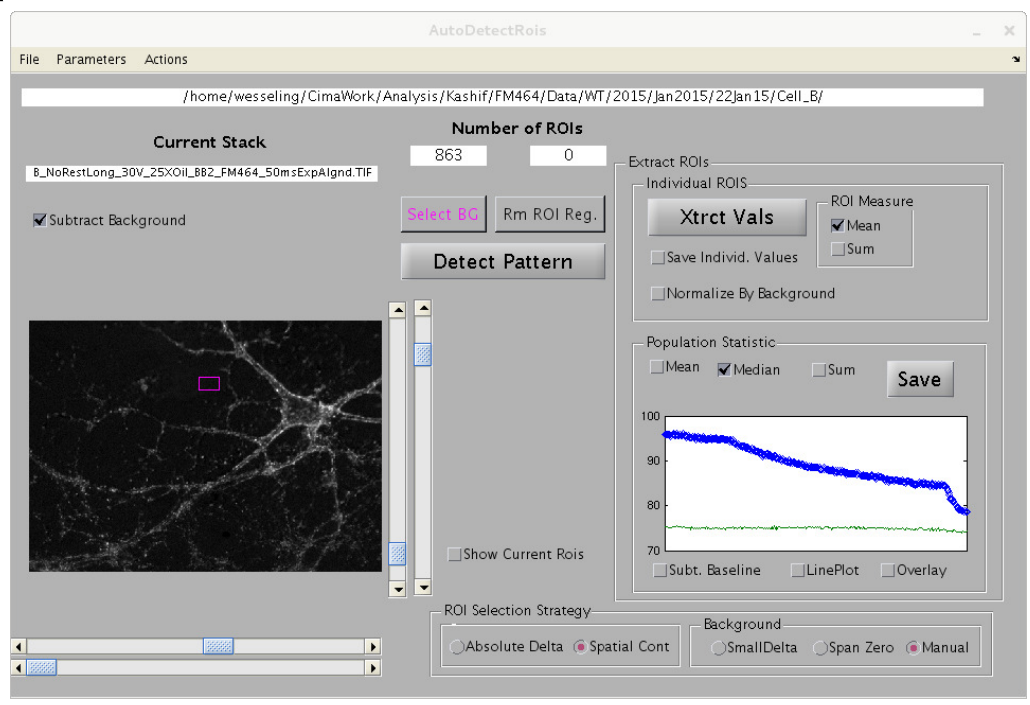

B

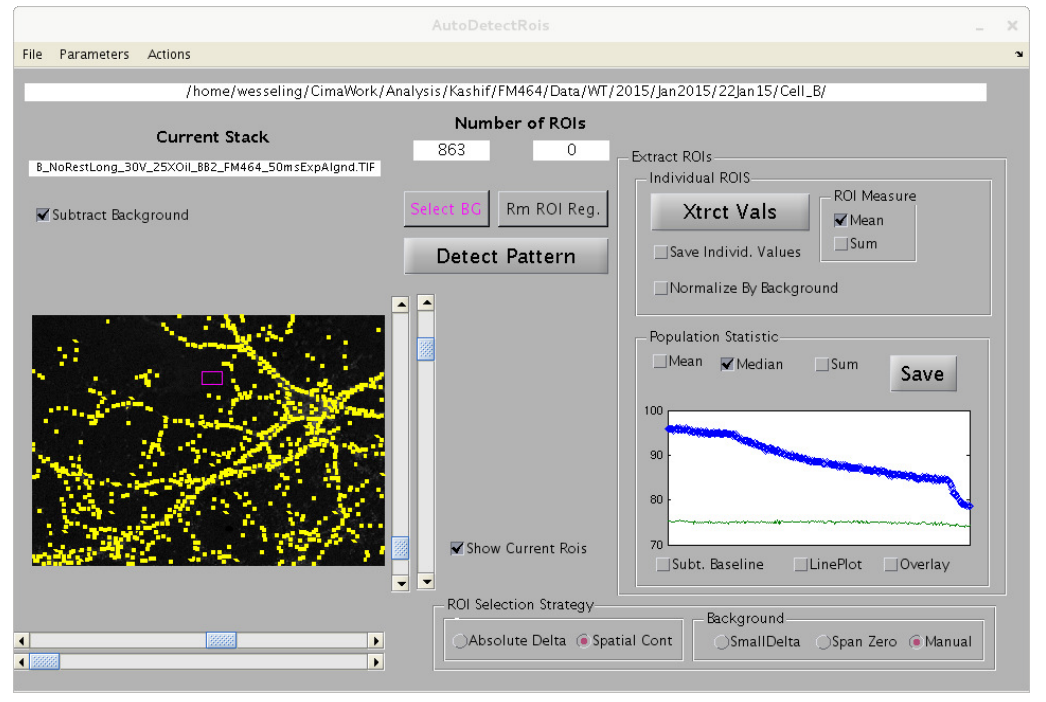

Supplementary Figure 3. Graphical user interface for semi-automatic ROI detection. A value for contrast for each 2 X2 pixel region of each image was calculated by subtracting the mean value of surrounding pixels from the mean value of the pixels within the region. Regions were then sorted by the change in contrast during the experiment, and regions overlapping with regions with larger change in contrast were eliminated. A threshold for the minimum change in contrast for consideration as a region of interest (ROI) was then set subjectively using the upper horizontal scroll bar beneath the image within the graphical user interface. The image is the difference calculated by subtracting the mean of destained images from the mean of images before destaining. The software has the capability of selecting background regions automatically, but, for the present study, a background region was selected by hand (magenta box). The blue circles in the plot in the "Population Statistic" box are the median ROI values vs time, and the green line is background. (A) Without displaying locations of ROIs. (B) ROIs are demarcated by yellow boxes. 